\title{
Protocolos para evaluación de desempeño en equipos médicos
}

\author{
M. Correa ${ }^{\psi}$, M. P. Villalba, J. H. García \\ Grupo de Investigación en Bioinstrumentación e Ingeniería Clínica - GIBIC, Programa de \\ Bioingeniería, Facultad de Ingeniería, Universidad de Antioquia UdeA, Medellín, Colombia
}

\begin{abstract}
Resumen - Las instituciones prestadoras de servicios de salud deben disponer de un control de calidad para los equipos médicos que garantice total confiabilidad en su funcionamiento y resultados, logrando el cumplimiento de los requisitos establecidos por las especificaciones técnicas y normas. En este contexto, las pruebas de desempeño en equipos biomédicos evalúan el desempeño, funcionamiento y cumplimiento de especificaciones de estos para brindar información confiable sobre su funcionamiento. En este trabajo se desarrolla un protocolo de evaluación de equipos médicos que permite cuantificar su desempeño, para esto se tiene en cuenta la información general del equipo, su estado y recomendaciones del fabricante entre otros; obteniendo un protocolo que pueda ser aplicado a diferentes tipos de equipos y que brinde información confiable y objetiva, en función de todos los aspectos que involucran su funcionamiento.
\end{abstract}

Palabras Clave — Equipo biomédico, pruebas de desempeño, calibración, ingeniería clínica.

\section{Protocols for Performance Evaluation in Medical Equipment}

\begin{abstract}
Healthcare service providers must count on the availability of quality control for their medical equipment which will assure total reliability in its operation, as well as results, thus complying with technical specifications and standards. Within this study, a medical equipment evaluation protocol is developed to quantify said equipment performance. In this context, performance tests in biomedical equipment assess the performance, operation, and compliance of biomedical equipment specifications to provide reliable information on its performance. This paper develops a protocol of evaluation for medical equipment that enables the quantification of its performance. To achieve this, it takes into account the general information of the equipment, its condition, as well as, manufacturer recommendations, among others, obtaining a protocol that can be applied to different types of equipment and that provides reliable and objective information, in terms of all the aspects that involve its operation.
\end{abstract}

Keywords - Biomedical equipment, performance test, calibration, clinical engineering.

\section{PROTOCOLOS PARA AVALIAČ̃̃O DE DESEMPENHO EM EQUIPAMENTOS MÉDICOS}

Resumo - As instituições prestadoras de serviços de saúde devem dispor de um controle de qualidade para as equipas médicas que garanta total fiabilidade em seu funcionamento e resultados, conseguindo o cumprimento dos requisitos estabelecidos pelas

\% Dirección para correspondencia: marianacorreabioing@gmail.com

DOI: https://doi.org/10.24050/19099762.n22.2017.1185 
especificações técnicas e normas. Neste contexto, as provas de desempenho em equipas biomédicos avaliam o desempenho, funcionamento e cumprimento de especificações destes para brindar informação confiável sobre seu funcionamento. Neste trabalho desenvolve-se um protocolo de avaliação de equipamentos médicos que permite quantificar seu desempenho, para isto se tem em conta a informação geral do equipamento, seu estado e recomendações do fabricante entre outros; obtendo um protocolo que possa ser aplicado a diferentes tipos de equipamentos e que brinde informação confiável e objetiva, em função de todos os aspectos que envolvem seu funcionamento.

Palavras-chave - Equipamento biomédico, provas de desempenho, calibração, engenharia clínica.

\section{INTRODUCCIÓN}

Ne considera la "evaluación del desempeño" como Oel conjunto de actividades que pueden incluir mediciones y que aporta evidencia para evaluar el desempeño, equipos biomédicos; y siendo la tecnología una parte fundamental en la prestación de servicios en salud, su adecuada evaluación y gestión constituyen un tema crucial para contribuir al logro de la eficiencia en la atención médica y al acceso efectivo a los servicios de salud con calidad. Paralelo a esto, en el ámbito nacional e internacional existen normas y estándares que rigen y orientan la evaluación de dispositivos médicos, haciendo necesario la unificación de criterios para implementar un sistema práctico de evaluación de desempeño de esta tecnología.

Las estadísticas elaboradas en países desarrollados aseguran que el $21 \%$ de los accidentes hospitalarios son ocasionados por la tecnología médica [1]. Así mismo, un estudio realizado en una IPS, publicado en la American Journal of Preventive Medicine [2], muestra que los eventos adversos asociados a tecnología médica suceden más por el fallo de los dispositivos que por error en su uso. Es por esto, que la adecuada evaluación de estas tecnologías es de suma importancia, el desempeño de los equipos biomédicos puede ser evaluado desde diversos puntos de vista, en particular son de interés los aspectos técnico, clínico y económico. Para las instituciones de salud es de gran importancia conocer el estado físico y funcional que poseen sus equipos en relación con su capacidad para satisfacer las necesidades clínicas para las que fueron adquiridos y la conveniencia económica de su funcionamiento.

En Colombia el Ministerio de Salud y Protección Social, específicamente la Dirección de Medicamentos y Tecnologías en Salud, trabajan en definir los requisitos mínimos para realizar estas actividades, que pueden incluir procesos metrológicos, y pueden ser realizadas dentro del servicio de soporte técnico, el mantenimiento o en otro momento del ciclo de vida de los equipos biomédicos y que sean denominadas evaluaciones de desempeño [3].

Es por esto que evaluar el desempeño de los equipos biomédicos cuando están en uso, contribuye a la seguridad del paciente y se debe considerar como una herramienta de vigilancia. Sin embargo, en el campo de la
Ingeniería clínica son pocos los centros de evaluación de tecnologías que permiten realizar las pruebas necesarias que, según la normatividad existente, brinden un informe del funcionamiento del equipo y permitan tomar decisiones sobre este, factor que influye en efectos adversos hacia los pacientes.

Este trabajo tiene como objetivo el desarrollo y aplicación de un plan de evaluación de equipo biomédico que permita cuantificar su desempeño según la normatividad, ofreciendo información objetiva y veraz, en función de los parámetros que determinan su funcionamiento.

Se inicia con una revisión exhaustiva de las normas y procesos de pruebas aplicables $[4,5,6]$. Luego se realiza el diseño de las pruebas y formatos de registro y finalmente se realiza la aplicación de las pruebas y finalmente se diseñan los informes correspondientes.

\section{Metodología}

Como paso principal para desarrollar los protocolos de evaluación de desempeño, se establecieron los requisitos comunes para ésta, es decir, la información general que debe ser tenida en cuenta tal como pruebas mínimas a implementar según las normas [7], estado de los accesorios del equipo, recomendaciones del fabricante, entre otros. Se diseñaron las pruebas de tal forma que no dependa de un tipo de equipo en particular y que puedan ser evaluadas y aplicadas a diferentes tipos de equipos, garantizando el buen desempeño de éste.

Se agrupan las características generales del equipo y se proponen las pruebas aplicables a evaluar, las cuales se definen según el tipo de equipo y las funciones que este realiza, adicionalmente, se evalúa el equipo bajo sus condiciones de trabajo y especificaciones del fabricante por lo cual deben definirse pruebas específicas según modelo y rangos de operación basados en sus especificaciones técnicas $[8,9]$ como se ve en la Fig. 1.

Las pruebas específicas para cada equipo se definen de acuerdo a la selección de los siguientes ítems: 1. variables de medida del equipo, 2. intervalos de medida y de trabajo; 3. número de valores a medir y el número de repeticiones. 
Luego de implementar el protocolo y las pruebas aplicables generales y específicas al equipo médico, se analiza el estado y desempeño de este para generar finalmente un reporte que permita conocer los resultados obtenidos, información de quien ejecuta las pruebas, quien realiza su respectiva revisión. Este reporte otorga información cuantitativa y cualitativa sobre si los equipos analizados cumplen o no con la normatividad y funciones especificados por el fabricante [10].

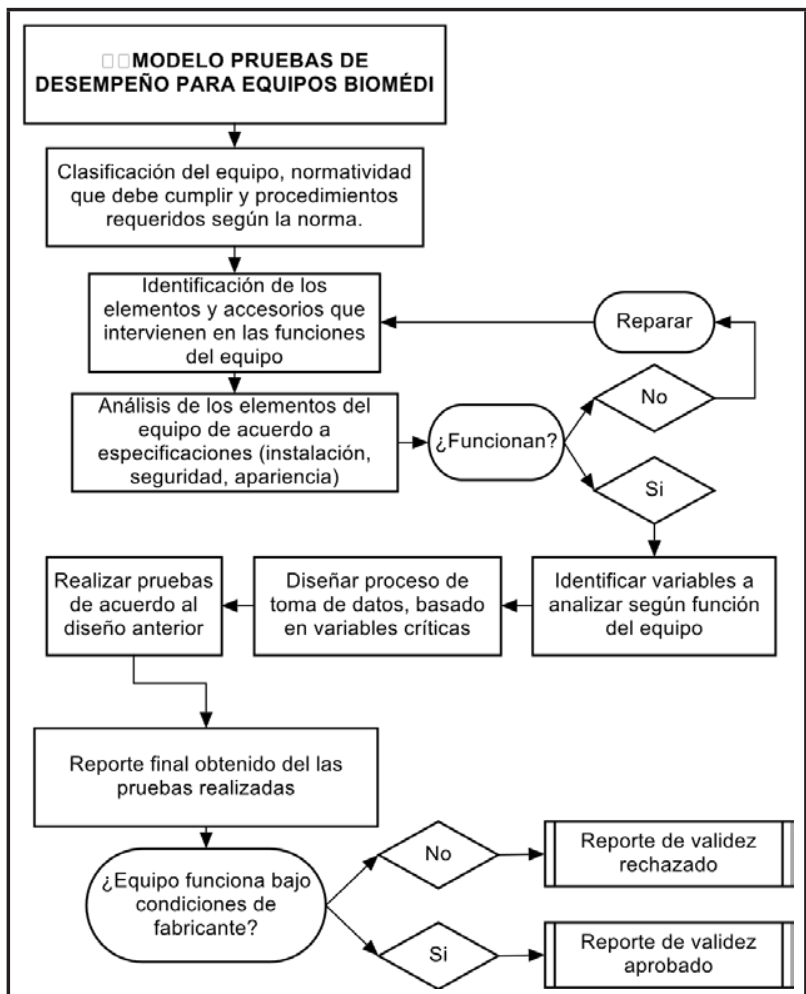

Fig 1. Esquema de metodología implementada. Fuente propia

El análisis del buen estado de los accesorios ligados al equipo médico, se realiza mediante las pruebas generales, las cuales incluyen evaluaciones de inspección visual y de seguridad eléctrica, a partir de éstas, se evalúa el funcionamiento de dichos accesorios y elementos complementarios de acuerdo a las especificaciones del fabricante y normas de seguridad estipuladas.

Confirmado el buen funcionamiento de todos los elementos implicados, se definen las variables relacionadas con el equipo médico y sus funciones, éstas son evaluadas mediante pruebas específicas por medio de mediciones, $\mathrm{y}$ procesos metrológicos para comparar los valores obtenidos con la norma respectiva y los permitidos por fábrica, los datos obtenidos son registrados en el protocolo para obtener un reporte que permita analizar el desempeño del equipo.
Finalmente, para cumplir la verificación del proceso de evaluación, se registra la firma tanto de quien realiza la evaluación, como de quien la revisa, este último es quien aprueba o rechaza la continua operación del equipo evaluado, bajo las condiciones anteriores.

\section{RESULTADOS}

\section{A. Formato General}

Se diseña un formato para implementar en diferentes equipos médicos, incluyendo identificación del equipo, sede, equipos necesarios para realizar las pruebas (patrones, insumos, formatos, manuales) y recomendaciones de seguridad; la segunda parte (Tabla 1); es la referente a pruebas generales según el tipo de equipo como el test de inspección visual, pruebas de estado y funcionamiento, se muestran solo algunos de los ítems en la tabla. Posteriormente se encuentran las pruebas específicas del equipo y características técnicas de este que se deben cumplir según el fabricante y finalmente la parte para incluir los resultados e informe final del equipo.

Tabla 1. Evaluación de desempeño, pruebas generales. Fuente propia

\begin{tabular}{|c|c|c|c|}
\hline \multicolumn{4}{|c|}{ PRUEBAS GENERALES } \\
\hline Característica & $\begin{array}{c}\text { Cumple según } \\
\text { especificaciones }\end{array}$ & si no & $\begin{array}{c}\text { valor/ } \\
\text { observaciones }\end{array}$ \\
\hline \multicolumn{4}{|l|}{$\begin{array}{l}\text { Seguridad } \\
\text { eléctrica }\end{array}$} \\
\hline \multirow{3}{*}{$\begin{array}{l}\text { Inspección } \\
\text { visual }\end{array}$} & $\begin{array}{l}\text { El dispositivo está } \\
\text { limpio }\end{array}$ & $\mathrm{X}$ & \\
\hline & $\begin{array}{l}\text { Sin daño físico en } \\
\text { la carcasa, pantalla, } \\
\text { perillas y } \\
\text { botones }\end{array}$ & $\mathrm{X}$ & \\
\hline & $\begin{array}{l}\text { Sin daño físico } \\
\text { en cable de ECG, } \\
\text { electrodos, sonda } \\
\text { de temperatura de } \\
\text { la piel, sensor de } \\
\text { SpO2 }\end{array}$ & $\mathrm{X}$ & $\begin{array}{l}\text { No se encuentra } \\
\text { cable de } \\
\text { oximetría }\end{array}$ \\
\hline
\end{tabular}

\section{B. Monitor de Signos Vitales}

La selección de los equipos bajo prueba (monitor de signos vitales a modo de ejemplo) se basó principalmente en su constante uso en los centros de salud y la importancia de su buen funcionamiento, además de cumplir funciones diferenciadas, lo cual resultó apropiado para el enfoque del proyecto.

Se analizaron las características genéricas de un monitor de signos vitales DRAGUER INFINITY DELTA XL y se establecieron las pruebas generales a realizar según dichas propiedades, como lo son prueba de seguridad eléctrica, inspección visual, funcionamiento y el estado de accesorios; para evaluar el equipo médico bajo condiciones 
de fábrica fue necesario disponer de la especificaciones técnicas del equipo con esto se diseñaron las pruebas específicas a evaluar tal como monitoreo de señales biológicas, alarmas sonaras, visuales y test de batería pruebas que se relacionan íntimamente con el funcionamiento y modelo del monitor de signos vitales en particular (Tabla 2).

Tabla 2. Evaluación de desempeño, pruebas específicas. Fuente propia

\begin{tabular}{|c|c|c|}
\hline \multicolumn{3}{|c|}{ PRUEBAS ESPECÍFICAS } \\
\hline Característica & $\begin{array}{l}\text { Cumple según } \\
\text { especificaciones }\end{array}$ & $\begin{array}{c}\text { valor/ } \\
\text { observaciones }\end{array}$ \\
\hline \multirow{2}{*}{ Batería } & $\begin{array}{c}\text { Carga de batería y duración } \\
\text { correctas }\end{array}$ & Sin cargador \\
\hline & $\begin{array}{c}\text { Mensajes de estado de carga } \\
\text { de la batería funcionales }\end{array}$ & Sin cargador \\
\hline \multirow{2}{*}{ Alarmas } & $\begin{array}{l}\text { Modificación de niveles de } \\
\text { alarma superior e inferior de } \\
\text { todos los parámetros medibles }\end{array}$ & \\
\hline & $\begin{array}{l}\text { Opción para silenciar alarma } \\
\text { funcional }\end{array}$ & \\
\hline \multirow{2}{*}{$\begin{array}{l}\text { Monitoreo de } \\
\text { ecg }\end{array}$} & $\begin{array}{l}\text { Despliegue simultaneo según } \\
\text { derivaciones }\end{array}$ & \\
\hline & $\begin{array}{l}\text { Modificación de canales y } \\
\text { derivaciones funcional }\end{array}$ & \\
\hline \multirow{2}{*}{$\begin{array}{l}\text { MONITOREO } \\
\text { spo } 2\end{array}$} & $\begin{array}{l}\text { Curva pletismografa } \\
\text { consistente }\end{array}$ & \\
\hline & Valores medidos* & \\
\hline \multirow{2}{*}{$\begin{array}{l}\text { Monitoreo } \\
\text { respiración }\end{array}$} & $\begin{array}{l}\text { Curva de respiración } \\
\text { consistente }\end{array}$ & \\
\hline & $\begin{array}{l}\text { Modificación de tamaño y } \\
\text { posición de la señal }\end{array}$ & \\
\hline \multirow{2}{*}{$\begin{array}{l}\text { Monitoreo } \\
\text { temperatura }\end{array}$} & $\begin{array}{l}\text { Canales de temperatura } \\
\text { consistentes }\end{array}$ & \\
\hline & $\begin{array}{l}\text { Despliegue numérico de } \\
\text { temperatura }\end{array}$ & \\
\hline $\begin{array}{l}\text { Monitoreo } \\
\text { presión no } \\
\text { invasiva }\end{array}$ & $\begin{array}{c}\text { Despliegue numérico de } \\
\text { presión (sistólica, diastólica } \\
\text { y media) }\end{array}$ & \\
\hline \multirow{2}{*}{$\begin{array}{l}\text { Mensajes e } \\
\text { indicadores de } \\
\text { estado }\end{array}$} & $\begin{array}{l}\text { Modificación de volumen de } \\
\text { mensajes audibles funcional }\end{array}$ & \\
\hline & $\begin{array}{l}\text { Indicadores visuales de estado } \\
\text { funcional }\end{array}$ & \\
\hline
\end{tabular}

En el protocolo mostrado se realizan 37 pruebas, se incluyen aquí solo dos por cada ítem a modo de ejemplo, las demás pruebas corresponden a las mediciones y eva- luación de las variables implicadas en el funcionamiento del equipo y sus valores permitidos respectivamente.

Mediante las pruebas específicas, se evalúan las funciones particulares del equipo tales como alarmas y sus respectivas modificaciones disponibles, funcionamiento de batería y las variables que dan paso a la toma de mediciones, se presentan a continuación algunas de las pruebas descritas.

Se evaluaron variables como saturación de oxígeno, temperatura, latidos por minuto y presión sistólica, diastólica y media, de las cuales se obtienen las mediciones para continuar la evaluación.

Al aplicar las pruebas diseñadas y utilizando simuladores de señales biológicas se obtuvo los valores respectivos de cada variable evaluada en puntos críticos y valores normales de paciente, al analizar estos resultados y teniendo en cuenta si se encuentran dentro de los rangos de funcionamiento y límites de error permitido según el fabricante, se concluye si el monitor de signos vitales es apto para continuar en operación o no.

A continuación, se presentan algunas de las mediciones realizadas para cada variable con sus respectivos valores permitidos y error propuesto por fábrica, cabe aclarar que dichos valores son tomados de las especificaciones del fabricante y estas pueden variar para cada tipo de equipo.

Como se observa en la Tabla 3, se tomaron cuatro datos por cada valor evaluado, con un error permitido de $\pm 1^{\circ} \mathrm{C}$.

Tabla 3. Mediciones y valores de temperatura. Fuente propia

\begin{tabular}{|c|c|c|c|c|}
\hline $\begin{array}{c}\text { Temp } \\
\left({ }^{\circ} \mathrm{C}\right) \\
\text { Patrón }\end{array}$ & $\begin{array}{l}\text { DUT } \\
\left({ }^{\circ} \mathrm{C}\right)\end{array}$ & \multicolumn{2}{|c|}{$\begin{array}{c}\text { Especificaciones y promedio } \\
\text { obtenido }\end{array}$} & $\begin{array}{c}\text { Según } \\
\text { Especificaciones }\end{array}$ \\
\hline \multirow{4}{*}{20} & 21 & Error: $\pm{ }^{\circ} \mathrm{C}$ & 1 & \\
\hline & 20,9 & Valor min permitido & 19 & Cumple \\
\hline & 20,6 & Valor max permitido & 21 & Cumple \\
\hline & 20,9 & Promedio & 20,85 & \\
\hline \multirow{4}{*}{23} & 22,9 & Error: $\pm{ }^{\circ} \mathrm{C}$ & 1 & \\
\hline & 23,8 & Valor min permitido & 22 & Cumple \\
\hline & 23,8 & Valor max permitido & 24 & Cumple \\
\hline & 23,7 & Promedio & 23,55 & \\
\hline
\end{tabular}

En la Tabla 4, se muestran las mediciones realizadas de presión, de las cuales se tomaron tres datos del valor evaluado, además, se analizan las pruebas con un error permitido de $\pm 3 \mathrm{mmHg}$. 
Tabla 4. Mediciones y valores de presión. Fuente propia

\begin{tabular}{|c|c|c|c|c|}
\hline $\begin{array}{c}\text { Presión } \\
\text { (mmHg) } \\
\text { Patrón }\end{array}$ & $\begin{array}{c}\text { Presión } \\
\text { (mmHg) } \\
\text { EBP }\end{array}$ & \multicolumn{2}{|c|}{$\begin{array}{l}\text { Especificaciones y } \\
\text { promedio obtenido }\end{array}$} & $\begin{array}{c}\text { Según } \\
\text { Especificaciones }\end{array}$ \\
\hline \multirow{4}{*}{100} & 100 & Error: $\pm \mathrm{mmHg}$ & 3 & \\
\hline & 101 & Valor min pdo & 97 & Cumple \\
\hline & 102 & Valor max pdo & 103 & Cumple \\
\hline & & Promedio & 101 & \\
\hline \multirow{4}{*}{80} & 83 & Error: $\pm \mathrm{mmHg}$ & 3 & \\
\hline & 83 & Valor min pdo & 77 & Cumple \\
\hline & 83 & Valor max pdo & 83 & Cumple \\
\hline & & Promedio & 83 & \\
\hline \multirow{4}{*}{120} & 124 & Error: $\pm \mathrm{mmHg}$ & 3 & \\
\hline & 122 & Valor min pdo & 117 & Cumple \\
\hline & 123 & Valor max pdo & 123 & Cumple \\
\hline & & Promedio & 123 & \\
\hline \multirow{4}{*}{150} & 148 & Error: $\pm \mathrm{mmHg}$ & 3 & \\
\hline & 148 & Valor min pdo & 147 & Cumple \\
\hline & 149 & Valor max pdo & 153 & Cumple \\
\hline & & Promedio & 148,3 & \\
\hline (1) & & de FCG & & \\
\hline $\begin{array}{c}\text { Patrón } \\
\text { bpm } \\
\end{array}$ & Leída & \multicolumn{2}{|c|}{$\begin{array}{l}\text { Especificaciones y } \\
\text { promedio obtenido }\end{array}$} & $\begin{array}{c}\text { Según } \\
\text { especificaciones }\end{array}$ \\
\hline \multirow{4}{*}{30} & 30 & Error: \pm & 2 & \\
\hline & 30 & Valor min pdo & 28 & Cumple \\
\hline & 30 & Valor max pdo & 32 & Cumple \\
\hline & & Promedio & 30 & \\
\hline \multirow{4}{*}{80} & 80 & Error: \pm & 2 & \\
\hline & 80 & Valor min pdo & 78 & Cumple \\
\hline & 80 & Valor max pdo & 82 & Cumple \\
\hline & & Promedio & 80 & \\
\hline \multirow{4}{*}{120} & 120 & Error: \pm & 2 & \\
\hline & 120 & Valor min pdo & 118 & Cumple \\
\hline & 120 & Valor max pdo & 122 & Cumple \\
\hline & & Promedio & 120 & \\
\hline
\end{tabular}

Con los valores anteriores, se exponen los resultados obtenidos en la prueba de señales electrocardiográficas, las cuales, corresponden a tres mediciones para cada valor propuesto en el simulador de ECG, adicionalmente, el error sobre el valor medido de \pm 2 bpm, dadas las especificaciones técnicas del equipo bajo prueba.

Para analizar fácilmente los resultados anteriores, se realiza una gráfica que nos permita confirmar si los valores obtenidos se encuentran o no dentro del rango de error propuesto por el fabricante, se muestra a continuación la representación gráfica solo para la variable ECG.

De la Fig. 2 se observa que las tres mediciones realizadas por cada valor, coinciden y se encuentran dentro de los límites permitidos y estipulados por el fabricante.

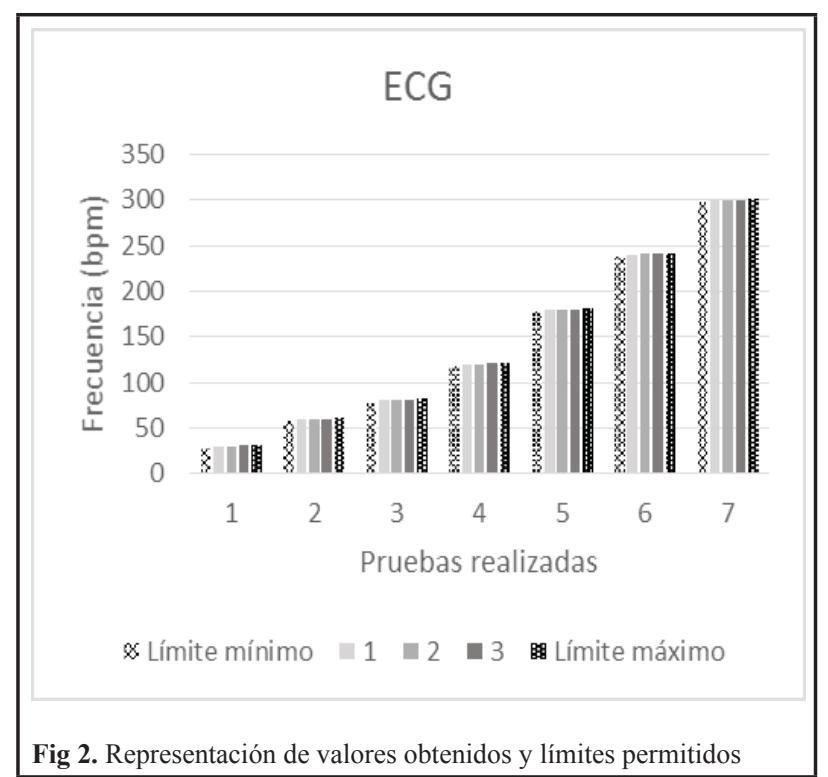

Gracias a los protocolos de evaluación diseñados y pruebas realizadas, se obtienen bases suficientes para tomar una decisión acerca del desempeño del equipo, las mostradas son un resumen de lo realizado, por lo cual no se expone el reporte completo de evaluación ni la decisión de validez final sobre el equipo evaluado.

\section{Discusión}

Los rangos de medición para la aplicación de las pruebas contribuyen notablemente a la evaluación de desempeño de los equipos, se considera necesario establecerlos teniendo en cuenta los valores generalmente utilizados y críticos en diagnóstico, puesto que son los que influyen en la valoración o procedimiento mientras el equipo médico se encuentra en operación.

Se deben tener en cuenta las observaciones obtenidas en las pruebas para determinar si el equipo puede continuar en funcionamiento, si es necesario establecer o no condiciones de uso y en caso afirmativo la justificación de estas, ya que el equipo médico puede presentar falencias mínimas y sin embargo no generar efectos adversos en el paciente ni atentar contra su seguridad.

La búsqueda constante de mejoras en la normatividad en pro de la seguridad del paciente ha llevado a ajustar los antiguos procesos de mantenimiento y calibración para 
obtener unos nuevos que ayuden a garantizar el correcto funcionamiento de los equipos médicos, todo esto a través de la redefinición de conceptos como lo es la forma de selección de equipos para calibración o control metrológico, o la aplicación de pruebas que permitan confirmar el funcionamiento de estos. La aplicación de las pruebas de desempeño es necesaria para cualquier equipo médico siendo una herramienta más para ayudar a garantizar el buen estado y funcionamiento de éste de acuerdo a sus especificaciones, contribuye a la toma de decisiones en la etapa de pre y pos mercado además de ayudar a futuros procesos de adquisición.

Los protocolos de evaluación del desempeño de tecnología propuestos apuntan a un cambio de paradigma en lo relacionado a los procedimientos e instructivos para la validación de estas tecnologías, puesto que posibilitan la certificación de la calidad de los productos garantizando que cumplen con la seguridad y exactitud correspondiente a los valores especificados por el fabricante.

Estos protocolos permiten la extrapolación a una gran cantidad de equipos de diferentes marcas, modelos, variables a medir y necesidades propias de cada institución, permitiendo realizar las pruebas adecuadas de acuerdo a cada tipo de tecnología o realizar agrupamiento por tipo de variable; por ejemplo diseñando pruebas de desempeño para electrocardiógrafos, monitores de presión arterial no invasiva, oxímetros que luego en conjunto corresponden a una prueba para monitor de signos vitales realizando pocas modificaciones al protocolo.

\section{CONCLusión}

Los protocolos propuestos durante el proyecto constituyen una base para realizar la evaluación de desempeño en los equipos especificados, permitiendo que con una adecuada selección de las características se puedan aplicar a tecnologías de diferentes marcas o modelos.

El principal insumo para la creación de estos protocolos son las indicaciones del fabricante, ya que rangos de medición, magnitudes fisiológicas, resolución, exactitud, entre otras; varían de un equipo a otro, o incluso de un modelo a otro de la misma marca.

Al diseñar un protocolo que abarca desde los puntos más generales hasta los más específicos por equipo, se puede contar con una guía bajo la cual es posible generar protocolos similares para muchos tipos de tecnología sin requerir de importantes cambios, lo que permite rápidamente ampliar el banco de protocolos de pruebas, siendo los mismos siempre sujetos de cambio y mejoras, pero al mismo tiempo permite llegar en poco tiempo a un protocolo estable que es fuente de información valiosa para la toma de decisiones o al mismo tiempo evidencia para futuros procesos de evaluación o adquisición de tecnología.

La validación de la funcionalidad de un dispositivo médico es importante para que su comercialización y uso no generen eventos adversos sobre el paciente, por lo que la creación de este tipo de protocolos es fundamental para la aceptación de las nuevas tecnologías o las ya existentes; sin embargo, se recomienda implementar mejoras en el diseño de las pruebas y estandarizar el protocolo creado para que pueda ser aplicable a cualquier tipo de equipo médico.

\section{Agradecimientos}

Este trabajo ha sido financiado por Sistema General de Regalías, Republica de Colombia a través del proyecto "Fortalecimiento de la plataforma tecnológica para la formación especializada en el área de la salud y el desarrollo de tecnología biomédica", código RutaN-139C. Los autores agradecen el apoyo brindado por el Hospital General de Medellín y el Laboratorio de Metrología Celsius Ltda.

\section{CONFLICTO DE INTERÉS}

Los autores declaran que no tienen conflicto de interés.

\section{REFERENCIAS}

[1]. "Cómo disminuir los accidentes en la atención de salud mediante calidad total, uso de computadora y otras medidas", Revista Latinoamericana de Derecho Médico y Medicina, no 7, pp. 43-54, 2003.

[2]. "Associated Adverse Events from Emergency Departments". American Journal of Preventive Medicine.

[3]. Ministerio de salud, "Guía Rápida para las mediciones en equipos biomédicos", [En línea]. Disponible en: https://www.minsalud. gov.co/sites/rid/Lists/Bibliot ecaDigital/RIDE/VS/MET/guiarapida-para-lasmediciones-en-equipos-biomedicos-v05282015. pdf. [Último acceso: 07 Octubre 2015].

[4]. Icontec, Equipos Electromédicos. Parte 1-2: Requisitos Generales de Seguridad. Norma Colateral. Compatibilidad Electromagnética. Requisitos y Ensayos. 2014, p. 10.

[5]. I. E. Comission, Medical electrical equipment - Part 249: Particular requirements for the basic safety and essential performance of multifunction patient monitoring equipment. 2011, p. 110.

[6]. IEC, Medical electrical equipment -- Part 1-8: General requirements for basic safety and essential performance -- Collateral standard: General requirements, tests and guidance for alarm systems in medical electrical equipment and medical electrical systems. 2006, p. 2012.

[7]. Normatividad para equipos y dispositivos médicos, INVIMA. [En línea]. Disponible en: https://www.invima.gov.co/images/pdf/tecnovigilan cia/ABC\%20Dispositivos\%20Medicos\%20INVIMA. pdf

[8]. Ministerio de Salud y Protección Social, Decreto 4725, por el cual se reglamenta el régimen de servicios sanitarios, permiso de co- 
mercialización y vigilancia sanitaria de los dispositivos médicos para uso humano. Colombia, 2005.

[9]. Instituto Colombiano de Normas Técnicas y Certificación, Norma Técnica Colombiana. NTC10012, Sistemas de gestión de la medición: requisitos para los procesos de medición y los equipos de medición. Colombia, 2003.

[10]. Ministerio de Salud y Protección Social, Decreto 2269, por el cual se organiza el sistema nacional de normalización, certificación y metrología. Colombia, 1993. 